\title{
CLIFFORD ALGEBRA-VALUED ORTHOGONAL POLYNOMIALS IN THE OPEN UNIT BALL OF EUCLIDEAN SPACE
}

\author{
FRED BRACKX, NELE DE SCHEPPER, and FRANK SOMMEN
}

Received 8 January 2004

\begin{abstract}
A new method for constructing Clifford algebra-valued orthogonal polynomials in the open unit ball of Euclidean space is presented. In earlier research, we only dealt with scalar-valued weight functions. Now the class of weight functions involved is enlarged to encompass Clifford algebra-valued functions. The method consists in transforming the orthogonality relation on the open unit ball into an orthogonality relation on the real axis by means of the so-called Clifford-Heaviside functions. Consequently, appropriate orthogonal polynomials on the real axis give rise to Clifford algebra-valued orthogonal polynomials in the unit ball. Three specific examples of such orthogonal polynomials in the unit ball are discussed, namely, the generalized Clifford-Jacobi polynomials, the generalized Clifford-Gegenbauer polynomials, and the shifted Clifford-Jacobi polynomials.
\end{abstract}

2000 Mathematics Subject Classification: 12E10, 30G35.

1. Introduction. In a series of papers [ $4,5,6,8,9]$, higher-dimensional wavelets and their corresponding continuous wavelet transforms have been studied in the framework of Clifford analysis. Clifford analysis, centred around the notion of monogenic functions, may be regarded as a direct and elegant generalization to higher dimensions of the theory of holomorphic functions in the complex plane. An essential step in the construction of these Clifford-wavelets is the introduction of specific polynomials satisfying orthogonality relations with respect to scalar-valued weight functions. These polynomials originate as a result of a particular Clifford analysis technique, the socalled Cauchy-Kowalewski extension of a real-analytic function in $\mathbb{R}^{m}$ to a monogenic function in $\mathbb{R}^{m+1}$. The Clifford-Hermite polynomials, the Clifford-Gegenbauer polynomials, and the Clifford-Laguerre polynomials constructed this way all give rise to wavelets in $\mathbb{R}^{m}$, since they all satisfy the necessary admissibility condition. For an account of the continuous wavelet transform in Clifford analysis and an overview of the generalized orthogonal polynomials and their corresponding wavelets thus far obtained, we refer the reader to [3].

In this paper, we develop a different method for constructing Clifford algebra-valued orthogonal polynomials in the open unit ball of Euclidean space. It should be emphasized that the class of weight functions, which up to now always were scalar-valued, is now enlarged to encompass Clifford algebra-valued real-analytic functions. In order to make the paper self-contained, a section on definitions and basic properties of Clifford algebra and Clifford analysis is included (see Section 2). In Section 3, the general method of our approach is presented. It consists, roughly speaking, of transforming 
the orthogonality relation on the open unit ball into an orthogonality relation on the real axis. Crucial to this transformation are the so-called Clifford-Heaviside functions; they generalize to higher dimensions the Heaviside step-function on the real axis and are a typical feature of Clifford analysis. Apparently our construction method is simple, but it should nevertheless be emphasized that this is entirely due to the power of Clifford analysis and the existence of these idempotent Clifford-Heaviside functions, inexistent in complex or harmonic analysis.

The method is then applied to three specific cases; in each of the cases known orthogonal polynomials on the interval $[-1,1]$ or $[0,1]$ lead to orthogonal Clifford algebravalued polynomials in the open unit ball. The obtained generalized Clifford-Jacobi polynomials (see Section 4) and shifted Clifford-Jacobi polynomials (see Section 7) are new; the generalized Clifford-Gegenbauer polynomials (see Section 6) coincide, up to constants, with the generalized Gegenbauer polynomials which were already introduced in [10], albeit in a different way. A number of those higher-dimensional orthogonal polynomials are explicitly calculated and in most cases an explicit recurrence relation is established.

2. Clifford algebra and Clifford analysis. Clifford analysis (see, e.g, [7, 11]) offers a function theory which is a higher-dimensional analogue of the theory of the holomorphic functions of one complex variable. The functions considered are defined in $\mathbb{R}^{m}(m>1)$ and take their values in the Clifford algebra $\mathbb{R}_{m}$ or its complexification $\mathbb{C}_{m}=\mathbb{R}_{m} \otimes \mathbb{C}$. If $\left(e_{1}, \ldots, e_{m}\right)$ is an orthonormal basis of $\mathbb{R}^{m}$, then a basis for the Clifford algebra $\mathbb{R}_{m}$ is given by $\left(e_{A}: A \subset\{1, \ldots, m\}\right)$ where $e_{\varnothing}=1$ is the identity element. The noncommutative multiplication in the Clifford algebra is governed by the rules

$$
\begin{gathered}
e_{j}^{2}=-1, \quad j=1, \ldots, m, \\
e_{j} e_{k}+e_{k} e_{j}=0, \quad j \neq k, j, k=1, \ldots, m .
\end{gathered}
$$

Conjugation is defined as the anti-involution for which

$$
\bar{e}_{j}=-e_{j}, \quad j=1, \ldots, m,
$$

with the additional rule $\bar{i}=-i$ in the case of $\mathbb{C}_{m}$.

For $k=0,1, \ldots, m$ fixed, we call

$$
\mathbb{C}_{m}^{k}=\left\{\sum_{\# A=k} a_{A} e_{A} ; a_{A} \in \mathbb{C}\right\}
$$

the subspace of $k$-vectors, that is, the space spanned by the products of $k$ different basis vectors.

The Euclidean space $\mathbb{R}^{m}$ is embedded in the Clifford algebras $\mathbb{R}_{m}$ and $\mathbb{C}_{m}$ by identifying $\left(x_{1}, \ldots, x_{m}\right)$ with the vector variable $\underline{x}$ given by

$$
\underline{x}=\sum_{j=1}^{m} e_{j} x_{j} .
$$


The product of two vectors splits up into a scalar part and a 2-vector or a so-called bivector part:

$$
\underline{x} \underline{y}=\underline{x} \cdot \underline{y}+\underline{x} \wedge \underline{y}
$$

where

$$
\begin{gathered}
\underline{x} \cdot \underline{y}=-\langle\underline{x}, \underline{y}\rangle=-\sum_{j=1}^{m} x_{j} y_{j}, \\
\underline{x} \wedge \underline{y}=\sum_{j=1}^{m} \sum_{k=j+1}^{m} e_{j} e_{k}\left(x_{j} y_{k}-x_{k} y_{j}\right) .
\end{gathered}
$$

In particular,

$$
\underline{x}^{2}=-\langle\underline{x}, \underline{x}\rangle=-|\underline{x}|^{2}
$$

In the sequel, the so-called Clifford-Heaviside functions

$$
P^{+}=\frac{1}{2}\left(1+i \frac{\underline{x}}{|\underline{x}|}\right), \quad P^{-}=\frac{1}{2}\left(1-i \frac{\underline{x}}{|\underline{x}|}\right)
$$

will play an important role; they were introduced independently by Sommen in [13] and McIntosh in [12].

Introducing spherical coordinates in $\mathbb{R}^{m}$ by

$$
\underline{x}=r \underline{\omega}, \quad r=|\underline{x}| \in\left[0,+\infty\left[, \underline{\omega} \in S^{m-1},\right.\right.
$$

with $S^{m-1}$ the unit sphere in $\mathbb{R}^{m}$, the Clifford-Heaviside functions can be rewritten as

$$
P^{+}=\frac{1}{2}(1+i \underline{\omega}), \quad P^{-}=\frac{1}{2}(1-i \underline{\omega}) .
$$

They are selfadjoint mutually orthogonal primitive idempotents

$$
P^{+}+P^{-}=1, \quad P^{+} P^{-}=P^{-} P^{+}=0, \quad\left(P^{+}\right)^{2}=P^{+}, \quad\left(P^{-}\right)^{2}=P^{-} .
$$

Furthermore, we have

$$
i \underline{\omega} P^{ \pm}= \pm P^{ \pm}
$$

and hence

$$
i \underline{x} P^{ \pm}= \pm r P^{ \pm}
$$

The central notion in Clifford analysis is the notion of monogenicity, the higher dimensional analogue of holomorphicity.

An $\mathbb{R}_{m}$ - or $\mathbb{C}_{m}$-valued function $F\left(x_{1}, \ldots, x_{m}\right)$ is called left monogenic in an open region of $\mathbb{R}^{m}$ if in that region

$$
\partial_{\underline{x}} F=0
$$


Here $\partial_{\underline{x}}$ is the Dirac operator in $\mathbb{R}^{m}$ :

$$
\partial_{\underline{x}}=\sum_{j=1}^{m} e_{j} \partial_{x_{j}}
$$

an elliptic vector differential operator of the first order, splitting the Laplacian

$$
\Delta_{m}=-\partial_{\underline{x}}^{2}
$$

The notion of right monogenicity is defined in a similar way by letting the Dirac operator act from the right.

Let $\Omega \subset \mathbb{R}^{m}$ be open, let $C$ be a compact orientable $m$-dimensional manifold with boundary $\partial C$, and define the oriented $\mathbb{R}_{m}$-valued surface element $d \sigma_{\underline{x}}$ on $\partial C$ by

$$
d \sigma_{\underline{x}}=\sum_{j=1}^{m}(-1)^{j} e_{j} d \hat{x}_{j}
$$

where

$$
d \hat{x}_{j}=d x_{1} \wedge \cdots \wedge\left[d x_{j}\right] \wedge \cdots \wedge d x_{m}, \quad j=1,2, \ldots, m
$$

If $n(\underline{x})$ stands for the outward pointing unit normal at $\underline{x} \in \partial C$, then

$$
d \sigma_{\underline{x}}=n(\underline{x}) d \Sigma(\underline{x})
$$

$d \Sigma(\underline{x})$ being the Lebesgue surface measure.

Suppose that $f \in C_{1}(\Omega)$ is right monogenic in $\Omega$. Then Cauchy's theorem states that, for each $C \subset \Omega$,

$$
\int_{\partial C} f(\underline{x}) d \sigma_{\underline{x}}=0
$$

An important particular example occurs in the following case: take $f=1$ and $C=$ $\bar{B}(1)=\left\{\underline{x} \in \mathbb{R}^{m} ;|\underline{x}| \leq 1\right\}$ the closed unit ball in $\mathbb{R}^{m}$. Then $\partial C=S^{m-1}$ and at each point $\underline{\omega} \in S^{m-1}, n(\underline{\omega})=\underline{\omega}$, whence $d \sigma_{\underline{\omega}}=\underline{\omega} d S(\underline{\omega})$ with $d S(\underline{\omega})$ the Lebesgue measure on $S^{m-1}$.

Consequently, we have

$$
\int_{S^{m-1}} \underline{\omega} d S(\underline{\omega})=0
$$

confirming the fact that $\underline{\omega}$ is a spherical harmonic.

The above result will be of crucial importance in our general method for constructing Clifford algebra-valued orthogonal polynomials in the open unit ball of $\mathbb{R}^{m}$.

3. The general construction method. In this section, we exhibit a general method for constructing Clifford algebra-valued polynomials of the form

$$
p_{n}(i \underline{x})=\sum_{k=0}^{n} a_{k}(i \underline{x})^{k}, \quad a_{k} \in \mathbb{C}, k=0,1,2, \ldots, n,
$$


which are orthogonal with respect to a Clifford algebra-valued weight function on the open unit ball $B(1)=\left\{\underline{x} \in \mathbb{R}^{m} ;|\underline{x}|<1\right\}$.

Note that the polynomials considered take their values in $\mathbb{C}_{m}^{0} \oplus \mathbb{C}_{m}^{1}$, that is, a scalar plus a vector, sometimes called a paravector.

DEFINITION 3.1. If $W(r)=\sum_{j=0}^{\infty} b_{j} r^{j}\left(b_{j} \in \mathbb{C}, j \in \mathbb{N}\right)$ is real-analytic in the neighbourhood of the origin $r=0$, then $W(i \underline{x})$ is defined as $W(i \underline{x})=\sum_{j=0}^{\infty} b_{j}(i \underline{x})^{j}$.

Proposition 3.2. If $W(r)$ is real-analytic in $]-\rho, \rho\left[\right.$, then, in $\stackrel{o}{B}(0, \rho):=\left\{\underline{x} \in \mathbb{R}^{m}\right.$ : $|\underline{x}|<\rho\}$,

(i) $W(i \underline{x})$ is real-analytic in the variables $\left(x_{1}, \ldots, x_{m}\right)$;

(ii) $W(i \underline{x}) P^{+}=P^{+} W(i \underline{x})=W(r) P^{+}$;

(iii) $W(i \underline{x}) P^{-}=P^{-} W(\underline{i x})=W(-r) P^{-}$;

(iv) $W(i \underline{x})=W(r) P^{+}+W(-r) P^{-}$.

Proof. (i) Straightforward.

(ii) Applying the properties of $P^{+}$, we have successively

$$
\begin{aligned}
W(\underline{i} \underline{x}) P^{+} & =\sum_{j=0}^{\infty} b_{j}(\underline{i x})^{j} P^{+}=\sum_{j=0}^{\infty} b_{j}(\underline{i \underline{x}})^{j}\left(P^{+}\right)^{j}=\sum_{j=0}^{\infty} b_{j}\left(r P^{+}\right)^{j} \\
& =\sum_{j=0}^{\infty} b_{j} r^{j} P^{+}=W(r) P^{+} .
\end{aligned}
$$

Moreover,

$$
W(\underline{i x}) P^{+}=P^{+} W(i \underline{x}) .
$$

(iii) Similar to (ii).

(iv) The formulae in (ii) and (iii) lead to

$$
W(i \underline{x})=W(i \underline{x}) P^{+}+W(i \underline{x}) P^{-}=W(r) P^{+}+W(-r) P^{-},
$$

where we have used the fact that $P^{+}+P^{-}=1$.

In what follows we will show how, by means of the above properties, integrals over the open unit ball $B(1)$ can be rewritten in terms of integrals over the real axis. Consequently, the problem of constructing Clifford algebra-valued polynomials $\left\{p_{n}(i \underline{x})\right\}_{n \geq 0}$ which are orthogonal on $B(1)$ will be reduced to constructing orthogonal polynomials on the real axis.

Two types of Clifford algebra-valued orthogonal polynomials on $B(1)$ will be distinguished.

3.1. Type 1. Orthogonality on $B(1)$ with respect to the weight function $W(i \underline{x})$. First, we consider the construction of Clifford algebra-valued orthogonal polynomials $\left\{p_{n}(i \underline{x})\right\}_{n \geq 0}$ with respect to a Clifford algebra-valued weight function $W(i \underline{x})$ in the unit ball $B(1)$. 
Let $\mathcal{O}(i \underline{x})$ denote the space of real-analytic functions in $B(1)$ of type

$$
U(\underline{i \underline{x}})=\sum_{j=0}^{\infty} c_{j}(\underline{i \underline{x}})^{j}, \quad c_{j} \in \mathbb{C},
$$

for which

$$
\int_{B(1)}|U(i \underline{x})|^{2} W(i \underline{x}) d \underline{x}<\infty,
$$

where the weight function $W(i \underline{x}) \in \mathcal{O}(i \underline{x})$ is fixed. Here $d \underline{x}$ stands for the Lebesgue measure on $B(1)$.

Then for any $U(i \underline{x}), V(i \underline{x}) \in \mathcal{O}(i \underline{x})$, we introduce the inner product

$$
(U(i \underline{x}), V(i \underline{x}))=\int_{B(1)} \bar{U}(\underline{i x}) V(i \underline{x}) W(i \underline{x}) d \underline{x} .
$$

By means of the above-mentioned properties of the idempotents $P^{+}$and $P^{-}$, this inner product is turned into

$$
\begin{aligned}
&(U(\underline{i} \underline{x}), V(i \underline{x})) \\
&=\int_{B(1)} \bar{U}(i \underline{x}) V(i \underline{x}) W(i \underline{x}) P^{+} d \underline{x}+\int_{B(1)} \bar{U}(i \underline{x}) V(i \underline{x}) W(i \underline{x}) P^{-} d \underline{x} \\
&= \int_{B(1)} \bar{U}(r) V(r) W(r) P^{+} d \underline{x}+\int_{B(1)} \bar{U}(-r) V(-r) W(-r) P^{-} d \underline{x} \\
&= \int_{0}^{1} \bar{U}(r) V(r) W(r) r^{m-1} d r \int_{S^{m-1}} \frac{1}{2}(1+i \underline{\omega}) d S(\underline{\omega}) \\
&+\int_{0}^{1} \bar{U}(-r) V(-r) W(-r) r^{m-1} d r \int_{S^{m-1}} \frac{1}{2}(1-i \underline{\omega}) d S(\underline{\omega}) \\
&= \frac{A_{m}}{2}\left(\int_{0}^{1} \bar{U}(r) V(r) W(r) r^{m-1} d r+\int_{0}^{1} \bar{U}(-r) V(-r) W(-r) r^{m-1} d r\right) \\
&= \frac{A_{m}}{2}\left(\int_{0}^{1} \bar{U}(r) V(r) W(r)|r|^{m-1} d r+\int_{-1}^{0} \bar{U}(u) V(u) W(u)|u|^{m-1} d u\right) \\
&= \frac{A_{m}}{2} \int_{-1}^{1} \bar{U}(r) V(r) W(r)|r|^{m-1} d r \\
&=(U(r), V(r))_{L_{2}(d \mu) .}
\end{aligned}
$$

Here $A_{m}$ denotes the area of the unit sphere $S^{m-1}$ in $\mathbb{R}^{m}$ and $d \mu=A_{m} / 2 W(r)|r|^{m-1} d r$.

So a function $U(i \underline{x}) \in \mathcal{O}(i \underline{x})$ is square integrable with respect to the weight function $W(i \underline{x})$ if and only if $U(r)$ is square integrable with respect to $d \mu$.

Now the polynomials $\left\{p_{n}(\underline{i} \underline{x})\right\}_{n \geq 0}$ will form an orthogonal system if, for $n \neq n^{\prime}$,

$$
\left(p_{n}(i \underline{x}), p_{n^{\prime}}(i \underline{x})\right)=0
$$

or, equivalently,

$$
\left(p_{n}(r), p_{n^{\prime}}(r)\right)_{L_{2}(d \mu)}=0 .
$$


So we may conclude that the polynomials $\left\{p_{n}(i \underline{x})\right\}_{n \geq 0}$ are orthogonal on $B(1)$ with respect to $W(i \underline{x})$ if and only if the polynomials $\left\{p_{n}(r)\right\}_{n \geq 0}$ are orthogonal on $[-1,1]$ with respect to the weight function $W(r)|r|^{m-1}$.

Note that in the special case where the dimension $m$ is odd, the polynomials $\left\{p_{n}(r)\right\}_{n \geq 0}$ should be orthogonal on $[-1,1]$ with respect to the weight function $W(r) r^{m-1}$.

3.2. Type 2. Orthogonality on $B(1)$ with respect to the weight function $W(i \underline{x}) P^{+}$. Now we search for Clifford algebra-valued polynomials $\left\{p_{n}(i \underline{x})\right\}_{n \geq 0}$ which are orthogonal on $B(1)$ with respect to a Clifford algebra-valued weight function of the form $W(i \underline{x}) P^{+}=W(r) P^{+}$, thus satisfying an orthogonality relation with respect to the inner product

$$
(U(i \underline{x}), V(i \underline{x}))=\int_{B(1)} \bar{U}(i \underline{x}) V(i \underline{x}) W(i \underline{x}) P^{+} d \underline{x}
$$

which can be rewritten as

$$
\begin{aligned}
(U(\underline{i \underline{x})}, V(\underline{i \underline{x}})) & =\int_{B(1)} \bar{U}(r) V(r) W(r) P^{+} d \underline{x} \\
& =\int_{0}^{1} \bar{U}(r) V(r) W(r) r^{m-1} d r \int_{S^{m-1}} \frac{1}{2}(1+i \underline{\omega}) d S(\underline{\omega}) \\
& =\frac{A_{m}}{2} \int_{0}^{1} \bar{U}(r) V(r) W(r) r^{m-1} d r \\
& =(U(r), V(r))_{L_{2}(d v)},
\end{aligned}
$$

where $d v=A_{m} / 2 W(r) r^{m-1} d r$.

Consequently, the polynomials $\left\{p_{n}(i \underline{x})\right\}_{n \geq 0}$ are orthogonal on $B(1)$ with respect to $W(i \underline{x}) P^{+}$if and only if the polynomials $\left\{p_{n}(r)\right\}_{n \geq 0}$ are orthogonal on $[0,1]$ with respect to the weight function $W(r) r^{m-1}$.

Note that when considering in $B(1)$ the weight function $W(i \underline{x}) P^{-}=W(-r) P^{-}$, one has, in a similar way as above,

$$
(U(i \underline{x}), V(i \underline{x}))=\frac{A_{m}}{2}(-1)^{m-1} \int_{-1}^{0} \bar{U}(r) V(r) W(r) r^{m-1} d r
$$

In this case, we thus need polynomials $\left\{p_{n}(r)\right\}_{n \geq 0}$ which are orthogonal on $[-1,0]$ with respect to the weight function $W(r) r^{m-1}$.

4. The generalized Clifford-Jacobi polynomials. In this section, we focus on Clifford algebra-valued orthogonal polynomials in $B(1)$ with respect to the specific Clifford algebra-valued weight function $(1+i \underline{x})^{\alpha}(1-i \underline{x})^{\beta}(\alpha, \beta>-1)$.

According to the general theory exposed in Section 3.1, we aim at orthogonal polynomials $\left\{p_{n}(r)\right\}_{n \geq 0}$ on $[-1,1]$ with respect to the weight function $(1+r)^{\alpha}(1-r)^{\beta}|r|^{m-1}$ $(\alpha, \beta>-1)$. 
In [14], Vanlessen considers the generalized Jacobi weight function

$$
w(x)=(1-x)^{\alpha}(1+x)^{\beta} h(x) \prod_{v=1}^{n_{0}}\left|x-x_{v}\right|^{2 \lambda_{v}}, \quad x \in[-1,1],
$$

where $n_{0}$ is a fixed number, with

$$
\begin{array}{ccc}
\alpha>-1, \quad \beta>-1, \quad & -1<x_{1}<x_{2}<\cdots<x_{n_{0}}<1, \\
-\frac{1}{2}<\lambda_{v}<0 & \text { or } \quad 0<\lambda_{v}<+\infty,
\end{array}
$$

and with $h$ real-analytic and strictly positive on $[-1,1]$.

This weight function leads to a sequence of orthogonal polynomials $\left\{P_{n}(x)\right\}_{n \geq 0}$, satisfying a recurrence relation; in [14], the asymptotic behaviour of the coefficients in this recurrence relation is studied. The generalized Jacobi weight function has been the subject of many other papers. However, neither an explicit expression, a Rodrigues formula, nor an explicit recurrence relation seems to exist for the polynomials $\left\{P_{n}(x)\right\}_{n \geq 0}$ associated to this weight function.

Now, putting $n_{0}=1, x_{1}=0,2 \lambda_{1}=m-1, h(x)=1$, and switching $\alpha$ and $\beta$ in (4.1), we obtain the specific weight function

$$
w^{*}(x)=(1+x)^{\alpha}(1-x)^{\beta}|x|^{m-1}, \quad x \in[-1,1] .
$$

Consequently, the weight function $(1+r)^{\alpha}(1-r)^{\beta}|r|^{m-1}(\alpha, \beta>-1)$ belongs to the class of generalized Jacobi weight functions, yielding the existence of a sequence of orthogonal polynomials $\left\{p_{n}(r)\right\}_{n \geq 0}$, which may be obtained by a Gram-Schmidt procedure starting from the set $\left\{1, r, r^{2}, \ldots\right\}$.

This eventually leads to a new sequence of Clifford algebra-valued orthogonal polynomials $\left\{J_{n}^{(\alpha, \beta)}(i \underline{x})\right\}_{n \geq 0}$ in the unit ball $B(1)$ with respect to the weight function $(1+$ $i \underline{x})^{\alpha}(1-i \underline{x})^{\beta}(\alpha, \beta>-1)$.

As a result of the above-mentioned Gram-Schmidt procedure, we find, for the lowerdegree polynomials,

$$
\begin{aligned}
J_{0}^{(\alpha, \beta)}(i \underline{x})= & 1, \\
J_{1}^{(\alpha, \beta)}(i \underline{x})= & (i \underline{x})-\frac{N(1)}{N(0)}, \\
J_{2}^{(\alpha, \beta)}(i \underline{x})= & (i \underline{x})^{2}-\frac{N(3) N(0)-N(1) N(2)}{N(2) N(0)-(N(1))^{2}}(i \underline{x})-\frac{N(2)}{N(0)} \\
& +\frac{N(3) N(0)-N(1) N(2)}{N(2) N(0)-(N(1))^{2}} \frac{N(1)}{N(0)},
\end{aligned}
$$

and so forth, where

$$
\begin{aligned}
N(n)= & (-1)^{n} B(n+m, \alpha+1) F(-\beta, n+m ; \alpha+n+m+1 ;-1) \\
& +B(n+m, \beta+1) F(-\alpha, n+m ; \beta+n+m+1 ;-1),
\end{aligned}
$$

with $B(x, y)$ the beta function and $F(a, b ; c ; z)$ the hypergeometric function.

We call these polynomials the generalized Clifford-Jacobi polynomials. 
5. Special case of the generalized Clifford-Jacobi polynomials. For the generalized Clifford-Jacobi polynomials constructed in the foregoing section, we have neither an explicit formula nor an explicit recurrence relation.

However, for the special case where $\beta=\alpha+1$, we do obtain an explicit recurrence relation. Indeed, in [1], Atia explicitly gives the coefficients in the recurrence relation of a sequence of monic orthogonal polynomials $\left\{P_{n}^{(\alpha, \alpha+1)}(x, \mu)\right\}_{n \geq 0}, \operatorname{Re}(\alpha)>-1$, on $[-1,1]$ with respect to the weight function $|x|^{-\mu}(1+x)^{\alpha}(1-x)^{\alpha+1}$. Here $\mu$ is an arbitrary parameter with $\operatorname{Re}(-\mu)>-1$. For $\mu=0$, one obtains the well-known Jacobi polynomial sequence.

The polynomials $\left\{P_{n}^{(\alpha, \alpha+1)}(x, \mu)\right\}_{n \geq 0}$ satisfy a three-term recurrence relation

$$
\begin{gathered}
P_{n+2}^{(\alpha, \alpha+1)}(x, \mu)=\left(x-\beta_{n+1}\right) P_{n+1}^{(\alpha, \alpha+1)}(x, \mu)-\gamma_{n+1} P_{n}^{(\alpha, \alpha+1)}(x, \mu), \\
P_{0}^{(\alpha, \alpha+1)}(x, \mu)=1, \quad P_{1}^{(\alpha, \alpha+1)}(x, \mu)=x-\beta_{0},
\end{gathered}
$$

with

$$
\begin{aligned}
\beta_{0} & =-\frac{\mu-1}{\mu-2 \alpha-3}, \\
\beta_{n+1} & =(-1)^{n} \frac{\mu(\mu-2 n-2 \alpha-4)+(-1)^{n+1}(2 \alpha+1)}{(2 n+2 \alpha+3-\mu)(2 n+2 \alpha+5-\mu)}, \\
\gamma_{2 n+1} & =2 \frac{(n+\alpha+1)(2 n+1-\mu)}{(4 n+2 \alpha+3-\mu)^{2}}, \\
\gamma_{2 n+2} & =\frac{(2 n+2)(2 n+2 \alpha+3-\mu)}{(4 n+2 \alpha+5-\mu)^{2}} .
\end{aligned}
$$

Putting $\mu=1-m$, we obtain orthogonal polynomials on $[-1,1]$ with respect to the weight function $|x|^{m-1}(1+x)^{\alpha}(1-x)^{\alpha+1}, \operatorname{Re}(\alpha)>-1$.

According to Section 3.1, the polynomials $\left\{P_{n}^{(\alpha, \alpha+1)}(x, 1-m)\right\}_{n \geq 0}$ lead in a straightforward manner to orthogonal Clifford algebra-valued polynomials $\left\{\tilde{J}_{n}^{(\alpha, \alpha+1)}(i \underline{x})\right\}_{n \geq 0}$ in $B(1)$ with respect to the Clifford-valued weight function $(1+i \underline{x})^{\alpha}(1-i \underline{x})^{\alpha+1} ; \alpha>-1$.

From (5.1), we obtain the recurrence relation they satisfy

$$
\begin{gathered}
\tilde{J}_{n+2}^{(\alpha, \alpha+1)}(i \underline{x})=\left(i \underline{x}-\tilde{\beta}_{n+1}\right) \tilde{J}_{n+1}^{(\alpha, \alpha+1)}(i \underline{x})-\tilde{\gamma}_{n+1} \tilde{J}_{n}^{(\alpha, \alpha+1)}(i \underline{x}), \quad n \geq 0, \\
\tilde{J}_{0}^{(\alpha, \alpha+1)}(i \underline{x})=1, \quad \tilde{J}_{1}^{(\alpha, \alpha+1)}(i \underline{x})=i \underline{x}-\tilde{\beta}_{0},
\end{gathered}
$$

with

$$
\begin{aligned}
\tilde{\beta}_{0} & =-\frac{m}{2 \alpha+m+2}, \\
\tilde{\beta}_{n+1} & =(-1)^{n} \frac{(m-1)(m+2 n+2 \alpha+3)+(-1)^{n+1}(2 \alpha+1)}{(2 n+2 \alpha+m+2)(2 n+2 \alpha+m+4)}, \\
\tilde{\gamma}_{2 n+1} & =2 \frac{(n+\alpha+1)(2 n+m)}{(4 n+2 \alpha+m+2)^{2}}, \\
\tilde{\gamma}_{2 n+2} & =\frac{(2 n+2)(2 n+2 \alpha+m+2)}{(4 n+2 \alpha+m+4)^{2}} .
\end{aligned}
$$


Using the symbolic software Maple, we get

$$
\begin{aligned}
\tilde{J}_{0}^{(\alpha, \alpha+1)}(i \underline{x}) & =1, \\
\tilde{J}_{1}^{(\alpha, \alpha+1)}(i \underline{x}) & =i \underline{x}+\frac{m}{2+m+2 \alpha}, \\
\tilde{J}_{2}^{(\alpha, \alpha+1)}(i \underline{x}) & =(i \underline{x})^{2}+\frac{2}{2 \alpha+4+m}(i \underline{x})-\frac{m}{2 \alpha+4+m}, \\
\tilde{J}_{3}^{(\alpha, \alpha+1)}(i \underline{x}) & =(i \underline{x})^{3}+\frac{2+m}{2 \alpha+6+m}(i \underline{x})^{2}-\frac{2+m}{2 \alpha+6+m}(i \underline{x}) \\
& -\frac{(2+m) m}{(2 \alpha+4+m)(2 \alpha+6+m)},
\end{aligned}
$$

and so forth. It can be verified, by a tedious calculation, that indeed the above polynomials coincide with the generalized Clifford-Jacobi polynomials of the foregoing section, where $\beta=\alpha+1$.

6. The generalized Clifford-Gegenbauer polynomials. In this section, we construct orthogonal Clifford algebra-valued polynomials in $B(1)$ with respect to the scalar-valued weight function $\left(1-|\underline{x}|^{2}\right)^{\alpha}, \alpha>-1$. These polynomials apparently are a special case of the generalized Clifford-Jacobi polynomials of Section 4 , where now $\alpha=\beta$.

The starting point is the generalized Gegenbauer orthogonal polynomials $\left\{S_{n}^{(\alpha, \gamma)}(x)\right\}_{n \geq 0}$ (see [2]) which are orthogonal on [-1,1] with respect to the weight function $\left(1-x^{2}\right)^{\alpha}|x|^{2 \gamma+1}, \alpha, \gamma>-1, \gamma \neq-1 / 2$.

Applying the general method of Section 3, we finally obtain the monic generalized Clifford-Gegenbauer polynomials $\left\{G_{n}^{(\alpha)}(i \underline{x})\right\}_{n \geq 0}$, which are orthogonal on $B(1)$ with respect to $\left(1-|\underline{x}|^{2}\right)^{\alpha}, \alpha>-1$.

Note however that already in [10] so-called generalized Gegenbauer polynomials $C_{n, m}^{\alpha}(\underline{x})$ were constructed by means of a Rodrigues formula.

Both sequences of Gegenbauer polynomials are related by

$$
\begin{aligned}
C_{2 n, m}^{\alpha}(\underline{x}) & =(-1)^{n} 2^{2 n}(\alpha+n+1)_{n}\left(\alpha+n+\frac{m}{2}\right)_{n} G_{2 n}^{(\alpha)}(i \underline{x}), \\
C_{2 n+1, m}^{\alpha}(\underline{x}) & =(-1)^{n} i 2^{2 n+1}(\alpha+n+1)_{n+1}\left(\alpha+n+\frac{m}{2}+1\right)_{n} G_{2 n+1}^{(\alpha)}(i \underline{x}),
\end{aligned}
$$

with $(a)_{n}$ the Pochhammer symbol.

7. The shifted Clifford-Jacobi polynomials. In order to construct Clifford algebravalued orthogonal polynomials in $B(1)$ with respect to the Clifford algebra-valued weight function $(1-i \underline{x})^{\alpha} P^{+}, \alpha>-1$, we need orthogonal polynomials on $[0,1]$ with respect to $(1-r)^{\alpha} r^{m-1}, \alpha>-1$ (see Section 3.2). These polynomials are obtained by the change of variables $x=2 r-1$ in the Jacobi polynomials $\left\{P_{n}^{(\alpha, m-1)}(x)\right\}_{n \geq 0}$, thus 
satisfying the orthogonality relation

$$
\begin{gathered}
\int_{0}^{1} P_{n}^{(\alpha, m-1)}(2 r-1) P_{n^{\prime}}^{(\alpha, m-1)}(2 r-1)(1-r)^{\alpha} r^{m-1} d r \\
=\frac{\Gamma(\alpha+n+1) \Gamma(m+n)}{n ! \Gamma(\alpha+n+m)(\alpha+2 n+m)} \delta_{n, n^{\prime}} .
\end{gathered}
$$

According to the general theory of Section 3, this orthogonality relation leads to

$$
\begin{gathered}
\int_{B(1)} P_{n}^{(\alpha, m-1)}(2(i \underline{x})-1) P_{n^{\prime}}^{(\alpha, m-1)}(2(i \underline{x})-1)(1-i \underline{x})^{\alpha} P^{+} d \underline{x} \\
=\frac{A_{m}}{2} \frac{\Gamma(\alpha+n+1) \Gamma(m+n)}{n ! \Gamma(\alpha+n+m)(\alpha+2 n+m)} \delta_{n, n^{\prime}}
\end{gathered}
$$

expressing the orthogonality on $B(1)$ of the polynomials

$$
Q_{n}^{(\alpha)}(i \underline{x})=P_{n}^{(\alpha, m-1)}(2(i \underline{x})-1), \quad n \in \mathbb{N},
$$

which we call the shifted Clifford-Jacobi polynomials.

From the explicit expression for the Jacobi polynomials we obtain an explicit expression for our shifted Clifford-Jacobi polynomials:

$$
\begin{aligned}
Q_{n}^{(\alpha)}(i \underline{x}) & =\sum_{k=0}^{n}\left(\begin{array}{c}
n+\alpha \\
k
\end{array}\right)\left(\begin{array}{c}
n+m-1 \\
n-k
\end{array}\right)(i \underline{x})^{k}(i \underline{x}-1)^{n-k} \\
& =\frac{\Gamma(\alpha+n+1)}{n ! \Gamma(\alpha+m+n)} \sum_{k=0}^{n}\left(\begin{array}{l}
n \\
k
\end{array}\right) \frac{\Gamma(\alpha+m+n+k)}{\Gamma(\alpha+k+1)}(i \underline{x}-1)^{k} .
\end{aligned}
$$

Moreover, the recurrence relation for the Jacobi polynomials leads to the following three-term recurrence relation for the polynomials $Q_{n}^{(\alpha)}(i \underline{x})$ :

$$
\begin{aligned}
2 n(\alpha+ & m+n-1)(\alpha+m+2 n-3) Q_{n}^{(\alpha)}(i \underline{x}) \\
= & \left((\alpha+m+2 n-3)_{3}(2 i \underline{x}-1)+\left(\alpha^{2}-(m-1)^{2}\right)(\alpha+m+2 n-2)\right) \\
& \times Q_{n-1}^{(\alpha)}(i \underline{x})-2(\alpha+n-1)(m+n-2)(\alpha+m+2 n-1) Q_{n-2}^{(\alpha)}(i \underline{x}),
\end{aligned}
$$

with $n=2,3,4, \ldots$

\section{REFERENCES}

[1] M. J. Atia, An example of nonsymmetric semi-classical form of class $s=1$; generalization of a case of Jacobi sequence, Int. J. Math. Math. Sci. 24 (2000), no. 10, 673-689.

[2] S. Belmehdi, Generalized Gegenbauer orthogonal polynomials, J. Comput. Appl. Math. 133 (2001), no. 1-2, 195-205.

[3] F. Brackx, N. De Schepper, and F. Sommen, New multivariable polynomials and their associated continuous wavelet transform in the framework of Clifford analysis, submitted to Computational Methods and Function Theory.

[4] _ The bi-axial Clifford-Hermite continuous wavelet transform, J. Natur. Geom. 24 (2003), no. 1-2, 81-100.

[5] - The Clifford-Gegenbauer polynomials and the associated continuous wavelet transform, Integral Transform. Spec. Funct. 15 (2004), no. 5, 387-404. 
[6] _ The Clifford-Laguerre continuous wavelet transform, Bull. Belg. Math. Soc. Simon Stevin 11 (2004), no. 2, 201-215.

[7] F. Brackx, R. Delanghe, and F. Sommen, Clifford Analysis, Research Notes in Mathematics, vol. 76, Pitman (Advanced Publishing Program), Massachusetts, 1982.

[8] F. Brackx and F. Sommen, Clifford-Hermite wavelets in Euclidean space, J. Fourier Anal. Appl. 6 (2000), no. 3, 299-310.

[9] - The generalized Clifford-Hermite continuous wavelet transform, Adv. Appl. Clifford Algebras 11 (2001), no. 51, 219-231.

[10] J. Cnops, Orthogonal functions associated with the Dirac operator, Ph.D. thesis, Ghent University, Ghent, Belgium, 1989.

[11] R. Delanghe, F. Sommen, and V. Souček, Clifford Algebra and Spinor-Valued Functions. A Function Theory for the Dirac Operator, Mathematics and Its Applications, vol. 53, Kluwer Academic Publishers, Dordrecht, 1992.

[12] A. McIntosh, Clifford algebras, Fourier theory, singular integrals, and harmonic functions on Lipschitz domains, Clifford Algebras in Analysis and Related Topics (Fayetteville, Ark, 1993) (J. Ryan, ed.), Stud. Adv. Math., CRC Press, Florida, 1996, pp. 33-87.

[13] F. Sommen, Some connections between Clifford analysis and complex analysis, Complex Variables Theory Appl. 1 (1982), no. 1, 97-118.

[14] M. Vanlessen, Strong asymptotics of the recurrence coefficients of orthogonal polynomials associated to the generalized Jacobi weight, J. Approx. Theory 125 (2003), no. 2, 198-237.

Fred Brackx: Clifford Research Group, Department of Mathematical Analysis, Ghent University, Galglaan 2, 9000 Gent, Belgium

E-mail address: fb@cage.ugent . be

Nele De Schepper: Clifford Research Group, Department of Mathematical Analysis, Ghent University, Galglaan 2, 9000 Gent, Belgium

E-mail address: nds@cage.ugent.be

Frank Sommen: Clifford Research Group, Department of Mathematical Analysis, Ghent University, Galglaan 2, 9000 Gent, Belgium

E-mail address: fs@cage.ugent.be 


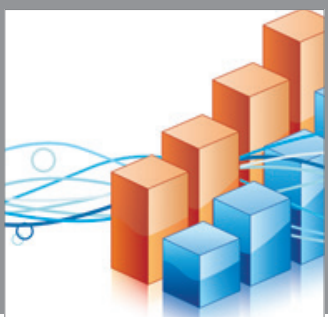

Advances in

Operations Research

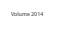

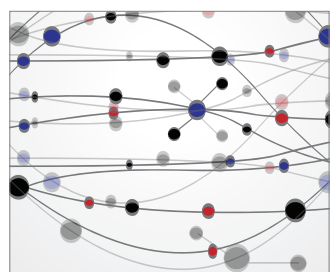

\section{The Scientific} World Journal
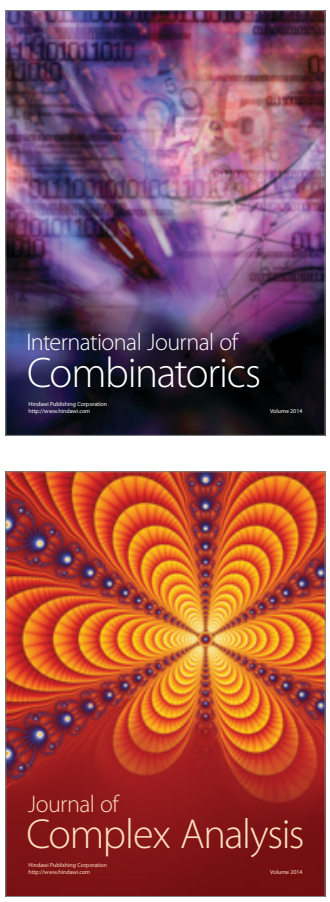

International Journal of

Mathematics and

Mathematical

Sciences
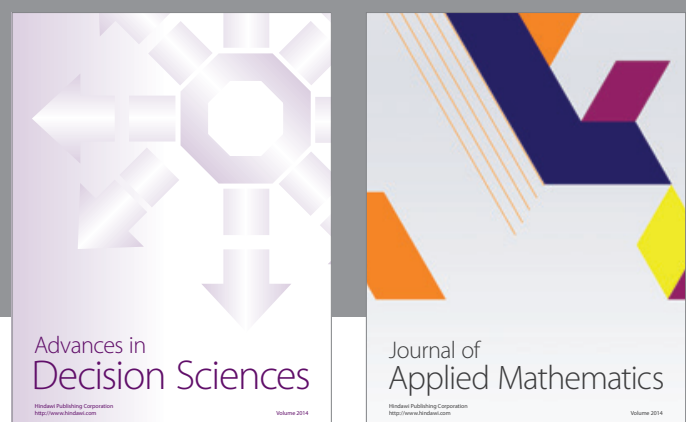

Journal of

Applied Mathematics
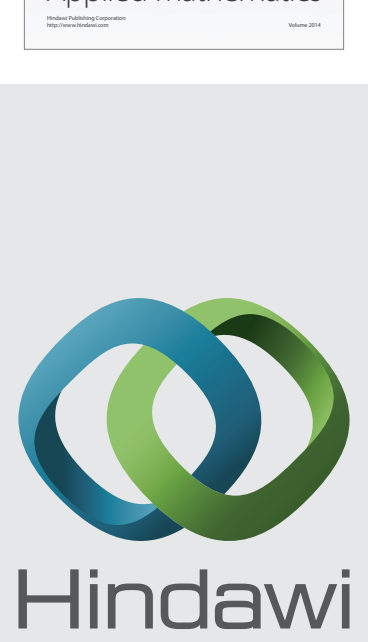

Submit your manuscripts at http://www.hindawi.com
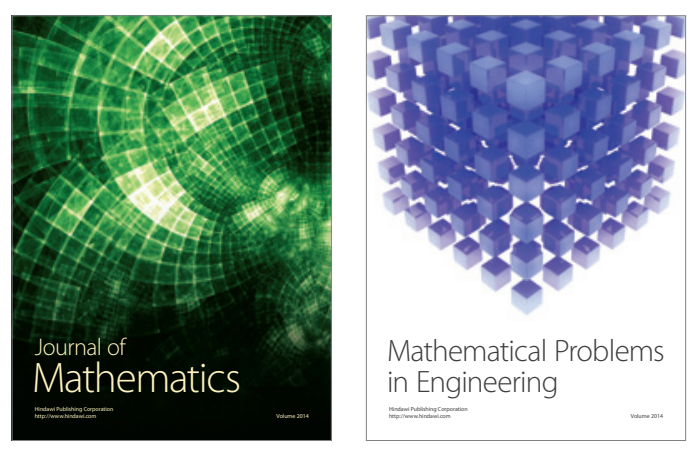

Mathematical Problems in Engineering
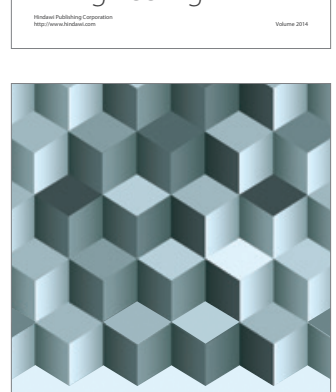

Journal of

Function Spaces
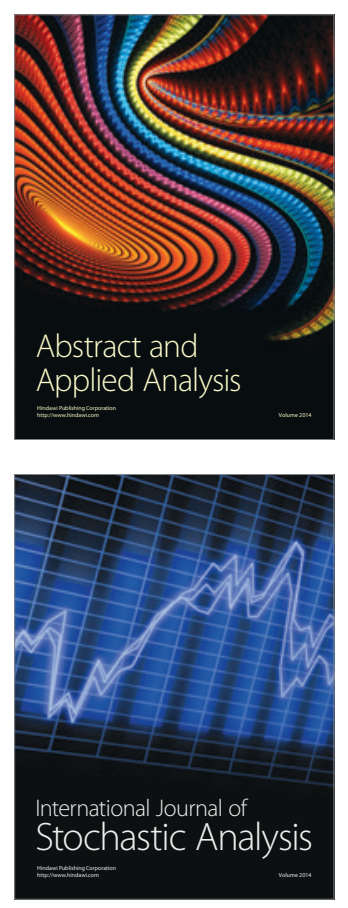

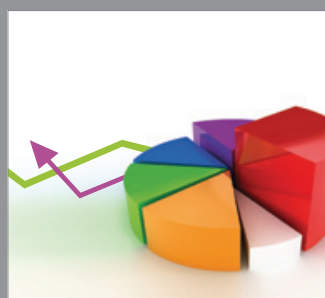

ournal of

Probability and Statistics

Promensencen
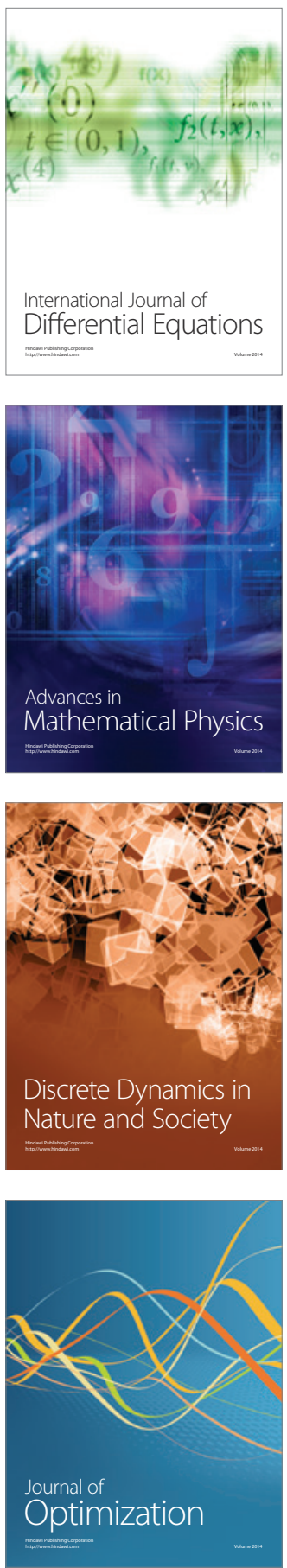